\title{
Reducing the Individual, Institutional and Societal Harms from Student Drug Use
}

\author{
Samantha Dick ${ }^{1}$, Brian Dillon ${ }^{2}$, Vasilis S. Vasiliou ${ }^{3}$, Martin P. Davoren ${ }^{1,4}$, Samantha \\ Dockray $^{3}$, Ciara Heavin ${ }^{2}$, Conor Linehan ${ }^{3}$, Michael Byrne ${ }^{5}$ \\ ${ }^{1}$ School of Public Health, University College Cork, Ireland, ${ }^{2}$ Health Information Systems \\ Research Centre, Cork University Business School, University College Cork, Ireland, \\ ${ }^{3}$ School of Applied Psychology, University College Cork, Ireland, ${ }^{4}$ Sexual Health Centre, \\ Cork, Ireland. ${ }^{5}$ Student Health Department, University College Cork, Ireland.
}

\begin{abstract}
Drug use among higher education students can cause harm to the individual, their institution, and the wider society. Academic performance, physical and mental health, institutional reputation, crime and unemployment can all be impacted by student drug use. Tackling this is a challenge, and is often compounded by limited student health and counselling capacity and the student's reluctance or unwillingness to seek support. Digital brief interventions have shown promise in reducing harm from substance use, and provide an opportunity to meet students where they are, delivering always-on, confidential support and intervention. However, limited interventions for drug use are available for students, and many struggle with engagement and retention. Our team have developed a novel brief intervention, using best practices in digital intervention development, and behavioural change to overcome some of these challenges. We describe the development of our intervention and discuss how implementation could result in tangible benefits to the individual, institution, and society.
\end{abstract}

Keywords: Drug use; digital intervention; harm reduction; behaviour change. 


\section{Introduction}

Drug use among higher education (HE) students is an under-researched and under-resourced area. Anecdotal evidence from student health departments and student welfare offices speak to an increasing prevalence of drug-related issues among students, but empirical data to back this up is limited. A recent survey of 18-25 year-olds in Ireland suggests that $50 \%$ are at risk of problems from drug use (Dooley et al., 2019). Further, around a quarter of students report recent drug use (Schulenberg et al., 2018). Drug use has been linked to a myriad of personal, institutional and societal harms, as illustrated in Figure 1.
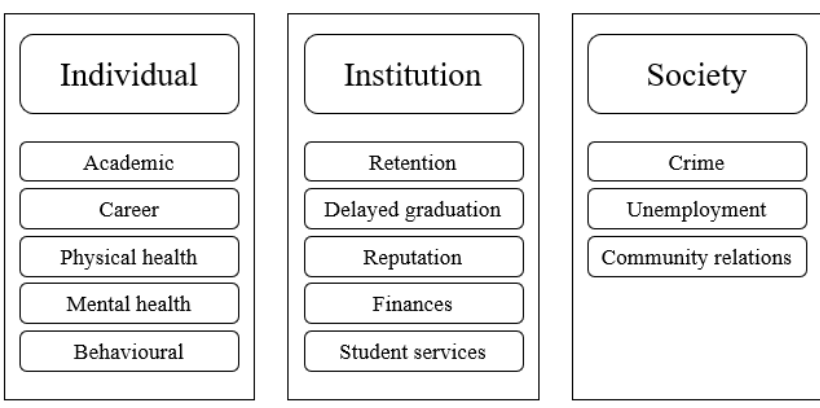

Figure 1. Individual, Institutional and Societal Drug-Related Harms

\subsection{Individual}

Harms from drug use are most concentrated to the individual and can include a range of academic challenges from missing classes (Kunst \& Gebhardt, 2018) and receiving lower grades (Arria et al., 2015), to failure to attain a degree (Horwood et al., 2010) and expulsion or suspension (Palmer et al., 2012). In the longer term, harms can extend to post graduation and impact on career trajectories for graduates (A. M. Arria et al., 2013).

In terms of physical health, students have reported panic attacks, insomnia and sleep problems, nausea seizures, memory loss, unconsciousness, weight loss, loss of mobility, and teeth problems (T. H. Bennett \& Holloway, 2014; Kunst \& Gebhardt, 2018). Students have reported significant mental health problems (Skidmore et al., 2016), including a worsening of a pre-existing condition (NUS \& Release, 2018), psychosis, increased depressive symptoms, deliberate self-harm, suicidal ideation and suicide attempts (Juan et al., 2015).

Students who use drugs have reported engaging in risky sexual behaviour (Skidmore et al., 2016), being aggressive or violent, getting involved in fights (Palmer et al., 2012; Skidmore et al., 2016). Additional harms can include legal (Palmer et al., 2012; Skidmore et al., 2016) and financial issues, failing to fulfil role function and losing interest in activities (T. H. 
Bennett \& Holloway, 2014). Further, students have reported minor harms such as embarrassment and guilt (Palmer et al., 2012).

\subsection{Institutional}

Drug use among students has been linked to increasing drop-out rates, delayed graduation and disruptions in college enrolment (A. M. Arria et al., 2013; Suerken et al., 2016). As many as $25 \%$ of students reporting minimal drug use may fail to complete college, increasing to $40 \%$ in chronic users (Amelia M. Arria et al., 2013). Further, interruptions to college enrolment have been reported in students who experience or seek treatment for depressive symptoms, for which drug use is a risk factor (Amelia M. Arria et al., 2013).

Issues with retention and progression can impact on institutional reputation and rankings. This may have a knock-on effect on the financial health of the institution as in some systems, external funding is dependent on student retentions rates (Yorke \& Longden, 2004). Further, reputational damage could affect alumni donations (Downes, 2017). Parents often play a key role in deciding where their child attends college, and often provide significant financial support. Anti-social behaviour or crime committed on campus or attributed to students or staff can also damage the reputation, and impact on local perceptions of the institution. Direct financial costs from drug use can occur from the services required to support students, including student health costs, welfare officer time, and student counselling hours.

\subsection{Societal}

Students who use drugs have reported committing crimes such as driving while under the influence, violence and property damage, drug selling, anti-social behaviour and theft (T. Bennett \& Holloway, 2018). A UK study found that $14 \%$ of drug users had come into contact with the criminal justice system as a result of their use. The majority had been searched for drugs by police or security, a quarter had received a police caution, and $10 \%$ had been arrested. A small percentage had been fined or charged with possession (NUS \& Release, 2018). Further, drug involvement during college may also have significant adverse effects on post-college employment, possibly contributing to unemployment rates (A. M. Arria et al., 2013). HE Institutions (HEIs) often form a central landmark of towns and cities. As a result, anti-social behaviour and crime can also impact on those living locally and damage relationships within the community (Munro \& Livingston, 2012).

\section{Brief Intervention Therapy}

Screening and brief interventions aim to identify current or potential problems with substance use, and motivate those at risk to change their behaviour. They are generally short, ranging from 5-30 minutes and are a valuable tool for addressing problematic or risky substance use behaviour. They can also be used to motivate those with more serious problems to seek and 
accept more intensive intervention, or serve as a signpost to services (Henry-Edwards et al. 2003). The digital delivery of brief interventions allows for greater accessibility, flexibility and confidentiality. Participants can access digital interventions anywhere, at any time. They can circumvent the limited resources of face-to-face care, and research indicates that they are as effective as clinician-delivered interventions (Hoch et al. 2016).

Digital brief interventions have shown success in reducing harm from alcohol and tobacco among college students. The most widely-used digital brief intervention tool in HE is eCheck Up to Go (e-CHUG), a brief assessment and personalised normative feedback tool developed in the US. e-CHUG has proven efficacy in reducing harm from alcohol and has been implemented in 600 HEIs (San Diego State University Research Foundation, 2018). There are far fewer interventions for drug use, but evidence suggests a moderate reduction in measures of harm (Dick et al., 2019). However, many existing interventions report issues with retention and engagement, and lack a theory-driven behaviour change framework. Further, many of the interventions found in the literature do not appear to be publicly available. Of those available, the majority have been developed for US-based HEIs, which have traditionally different cultures from European HEIs.

\section{Research and Development}

My Understanding of Substance-use Experiences (MyUSE) is a research and development project being carried out in a large university in Ireland. This project is led by a multidisciplinary team contributing expertise in student health, health information systems, public health and health psychology. The aim of the project is to develop and evaluate a digital behaviour change intervention for drug use among higher education students. We attempted to improve on the shortcomings of previous interventions by following a rigorous usercentred design methodology, in order to develop an engaging tool that students will want to use. Further, the content was developed using a well-established and robust methodology for intervention development following psychological theory. A Student Advisory Group were assembled at the outset of the project to guide the project from the perspective of the student, providing input and feedback on all aspects of the research, design, and development.

The first phase of the project was to research the area; establishing the efficacy of digital intervention for drug use, explore student motivations to change substance use behaviour, and establish best practices in intervention development. We conducted three systematic reviews to understand these key areas. Additionally, we conducted a survey of student drug use in the host institution, and conducted user-centred design workshops with students in order to better understand the issue of drug use, and how best to design our intervention to tackle it. 
The second phase of the project was the development and design stage. A synthesis of our previous research was undertaken, following the Behaviour Change Wheel methodology in order to identify specific clusters of behaviours to target. From this, a large number of behaviour change techniques (BCTs) were identified for inclusion in the intervention. The second part of this phase was then to translate these theory-ground BCTs into a digital format. The research and development teams worked closely to develop scripts and to devise appropriate digital presentations of the content. This work was underpinned by development of the technical infrastructure to support these features. Figure 2 presents an overview of the content development from behaviour cluster identification to digital implementation. Throughout the development, several workshops were conducted with students to test and validate the digital content and platform.
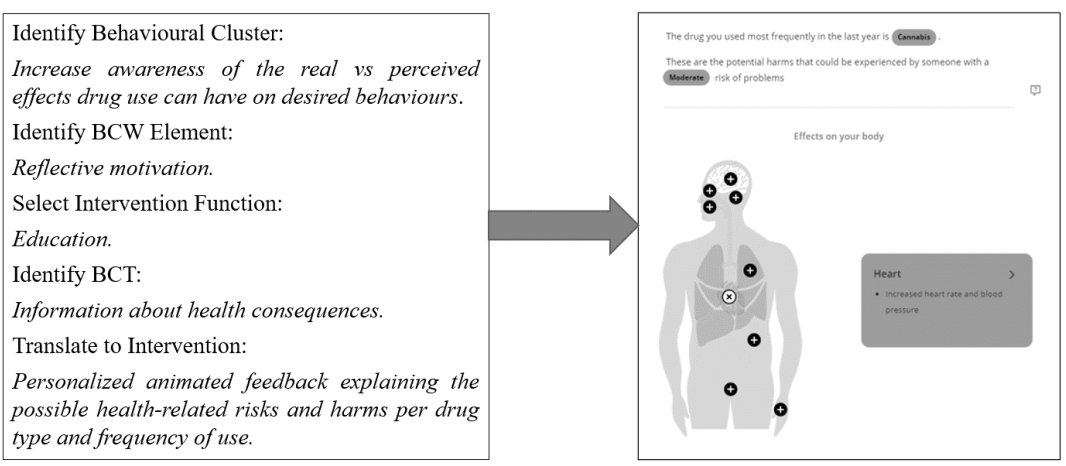

Figure 2. MyUSE Content Development.

MyUSE v1 was released in September 2020. This is a web and app-based intervention which allows students to self-assess their drug use and risk of problems using validated measures, receive personalised feedback on harms and education around drug use. Further, the intervention incorporates motivational interviewing, tailored behaviour change components and contextual skill-based activities, such as improving mindful decision making in relation to drug use behaviour.

The final phase is ongoing. Our aim is to evaluate the efficacy of the individual BCTs in order to optimise the intervention. We will achieve this using a fractional factorial evaluation, allowing us to assess a number of combinations of BCTs to determine the efficacy of each component. Following this, a final phase of development will take place to optimise the intervention, based on findings from the evaluation. We will retain the most efficacious components, and discard those which do not achieve the desired effects, resulting in a streamlined intervention. It is our aim to conduct a comprehensive technical, clinical and economic evaluation of the intervention in the future. 


\section{Potential Intervention Benefits}

A US study of student health attendances (Turner \& Keller, 2015) resulted in over 900,000 unique visits from 730,000 enrolled students per academic year, averaging 1.23 visits per student. Mental health accounted for $13 \%$ of these visits, and drug abuse accounted for $13 \%$ of mental health related visits. Mental health disorders had the highest number of visits per patient, at 4.93 on average. Extrapolating these figures to the Irish HEI context, based on a student population of 250,000 we can assume that there would be approximately 312,500 visits to student health services each academic year. Mental health would account for 60,625 and of these; 5,281 would be drug-abuse related.

This figure is likely to be conservative, given that the study above likely included only those with a clinically diagnosed drug abuse disorder. Further, most students declaring drug use will rarely visit traditional student health services, despite experiencing harms from their use (Schettino et al., 2015). The recent Irish study noted above reported that $50 \%$ of young people are at risk of problems from their drug use, suggesting that the need for support from college services could be higher. The precise impact and costs of drug use are difficult to quantify. However, the available research highlights potential for significant individual, institutional and societal harms, therefore measures should be taken to prevent them where possible.

In line with our research on other digital brief interventions, we would expect the implementation of the intervention to result in a correction of social norms and at least a small-medium reduction in harm from drug use (Dick et al., 2019). This could result in reduced harms to the individual, and at a population level, reduce the impact on the institution and society. MyUSE is built upon an intuitive reporting system based on user engagement. This data will also be used to inform the development of future intervention versions in conjunction with customer feedback. Meaningful inferences from the data are displayed within the administration area, which indicates user trends and engagement data. The administration area is a useful tool in identifying what issues may be a priority, as well as characterising local culture norms surrounding substance use. We expect that this data will contribute to a comprehensive national aggregate dataset of student drug use trends and behaviours and could be used to inform local and national policy.

\section{Conclusion}

MyUSE has been developed with the aim of implementing in HEIs across Ireland, the UK, Europe and beyond. The intervention provides an always-on, confidential service to students that they can access as and when they need, contributing to a reduction in the myriad of individual, institutional, and societal harms caused by drug use. Importantly, this intervention provides support to those students who may be experiencing harm from drug use, but are not motivated enough, or lack the awareness required to seek help and support. Further, it 
incorporates administrative control and data collection for each institution. The intervention will collect institution and national-level data on student drug use trends, harms and consequences, providing institutions with a comprehensive dataset which can be used to support student services and policy development.

\section{References}

Arria, A. M., Caldeira, K. M., Bugbee, B. A., Vincent, K. B., \& O’Grady, K. E. (2015). The Academic Consequences of Marijuana Use During College. Psychology of Addictive Behaviors, 29(3), 564-575. https://doi.org/DOI: 10.1037/adb0000108

Arria, A. M., Garnier-Dykstra, L. M., Cook, E. T., Caldeira, K. M., Vincent, K. B., Baron, R. A., \& O'Grady, K. E. (2013). Drug use patterns in young adulthood and post-college employment. Drug and Alcohol Dependence, 127(1-3), 23-30. https://doi.org/DOI: 10.1016/j.drugalcdep.2012.06.001

Arria, Amelia M., Caldeira, K. M., Vincent, K. B., Winick, E. R., Baron, R. A., \& O’Grady, K. E. (2013). Discontinuous College Enrollment: Associations With Substance Use and Mental Health. Psychiatric Services, 64(2), 165-172. https://doi.org/10.1176/appi.ps.201200106

Arria, Amelia M., Garnier-Dykstra, L. M., Caldeira, K. M., Vincent, K. B., Winick, E. R., \& O'Grady, K. E. (2013). Drug Use Patterns and Continuous Enrollment in College:Results From a Longitudinal Study. Journal of Studies on Alcohol and Drugs, 74(1), 71-83. https://doi.org/10.15288/jsad.2013.74.71

Bennett, T. H., \& Holloway, K. R. (2014). Drug Misuse Among University Students in the UK: Implications for Prevention. Substance Use \& Misuse, 49(4), 448-455. https://doi.org/DOI: 10.3109/10826084.2013.846378

Bennett, T., \& Holloway, K. (2018). Drug and Alcohol-Related Crime Among University Students. International Journal of Offender Therapy and Comparative Criminology, 62(14), 4489-4509. https://doi.org/10.1177/0306624X18769601

Dick, S., Whelan, E., Davoren, M. P., Dockray, S., Heavin, C., Linehan, C., \& Byrne, M. (2019). A systematic review of the effectiveness of digital interventions for illicit substance misuse harm reduction in third-level students. BMC Public Health, 19(1), 1244. https://doi.org/DOI: 10.1186/s12889-019-7583-6

Dooley, B., O’Connor, C., Fitzgerald, A., \& O’Reilly, A. (2019). My World Survey 2. University College Dublin; Jigsaw National Cenre for Youth Mental Health, Dublin.

Downes, M. (2017). University scandal, reputation and governance. International Journal for Educational Integrity, 13(1), 1-20. https://doi.org/10.1007/s40979-017-0019-0

Henry-Edwards, S., Humeniuk, R., Ali, R., Monteiro, M., \& Poznyak, V. (2003). Brief intervention for substance use: A manual for use in primary care. Draft Version, 1.

Hoch, E., Preuss, U. W., Ferri, M., \& Simon, R. (2016). Digital Interventions for Problematic Cannabis Users in Non-Clinical Settings: Findings from a Systematic Review and MetaAnalysis. European Addiction Research, 22(5), 233-242. https://doi.org/10.1159/000445716 
Horwood, L. J., Fergusson, D. M., Hayatbakhsh, M. R., Najman, J. M., Coffey, C., Patton, G. C., ... Hutchinson, D. M. (2010). Cannabis use and educational achievement: Findings from three Australasian cohort studies. Drug and Alcohol Dependence, 110(3), 247-253. https://doi.org/DOI: 10.1016/j.drugalcdep.2010.03.008

Juan, W., Jian-Xiong, D., Lan, G., Yuan, H., Xue, G., Jing-Hui, H., ... Ci-Yong, L. (2015). Non-medical use of psychoactive drugs in relation to suicide tendencies among Chinese adolescents. Addictive Behaviors, 51, 31-37. https://doi.org/DOI: 10.1016/j.addbeh.2015.07.003

Kunst, L. E., \& Gebhardt, W. A. (2018). Prevalence and Psychosocial Correlates of PartyDrug Use and Associated Problems among University Students in the Netherlands. Substance Use \& Misuse, 53(12), 2077-2088. https://doi.org/DOI: 10.1080/10826084.2018.1455700

Munro, M., \& Livingston, M. (2012). Student Impacts on Urban Neighbourhoods: Policy Approaches, Discourses and Dilemmas. Urban Studies, 49(8), 1679-1694.

NUS \& Release. (2018). Taking the Hit: Student Drug Use and How Institutions Respond. NUS London.

Palmer, R. S., McMahon, T. J., Moreggi, D. I., Rounsaville, B. J., \& Ball, S. A. (2012). College Student Drug Use: Patterns, Concerns, Consequences, and Interest in Intervention. Journal of College Student Development, 53(1), 124-132. https://doi.org/DOI: 10.1353/csd.2012.0014

San Diego State University Research Foundation. (2018). ECHECKUP TO GO Research. Retrieved 24 February 2021, from http://www.echeckuptogo.com/research

Schettino, J., Leuschner, F., Kasten, L., Tossmann, P., \& Hoch, E. (2015). Treatment of cannabis-related disorders in Europe (No. 929168760X). Publications Office of the European Union.

Schulenberg, J. E., Johnston, L. D., O’Malley, P. M., Bachman, J. G., Miech, R. A., \& Patrick, M. E. (2018). Monitoring the Future National Survey Results on Drug Use, 1975 2017. Volume II, College Students \& Adults Ages 19-55. Institute for Social Research.

Skidmore, C. R., Kaufman, E. A., \& Crowell, S. E. (2016). Substance use among college students. Child and Adolescent Psychiatric Clinics, 25(4), 735-753. https://doi.org/DOI: 10.1016/j.chc.2016.06.004

Suerken, C. K., Reboussin, B. A., Egan, K. L., Sutfin, E. L., Wagoner, K. G., Spangler, J., \& Wolfson, M. (2016). Marijuana use trajectories and academic outcomes among college students. Drug and Alcohol Dependence, 162, 137-145. https://doi.org/DOI: 10.1016/j.drugalcdep.2016.02.041

Turner, J. C., \& Keller, A. (2015). College Health Surveillance Network: Epidemiology and Health Care Utilization of College Students at US 4-Year Universities. Journal of American College Health, 63(8), 530-538. https://doi.org/10.1080/07448481.2015.1055567

Yorke, M., \& Longden, B. (2004). Retention and student success in higher education. McGraw-Hill Education (UK). 